\title{
Rehabilitación, apoyo social y atención comunitaria a personas con trastorno mental grave. Propuestas desde Andalucía
}

RESUMEN: Propuestas sobre terminología, estructura y funcionamiento de servicios de salud mental en aspectos como la atención comunitaria, rehabilitación y apoyo social. La situación concreta de Andalucía.

PALABRAS CLAVE: Atención comunitaria en salud mental, rehabilitación psicosocial, programas de apoyo social.
SUMMARY: Proposals concerning terms, structures and the functioning of mental health services in areas which include community care, rehabilitation and social support. The specific situation in Andalusia.

KEY WORDS: Community Mental Health, psychosocial rehabilitation, social support programmes.

La «rehabilitación»en salud mental sigue siendo un tema controvertido, tanto en su vertiente teórica como en la práctica. En la teórica porque, aunque probablemente ningún profesional del campo de la salud mental se negaría hoy a reconocer que la atención a personas con enfermedades mentales graves incluye componentes de rehabilitación, ese aparente acuerdo encubre, a menudo, discrepancias importantes cuyo significado real no siempre está bien precisado. Pero, además, ese reconocimiento teórico, cuando se da, no suele tener una repercusión práctica directa en relación con la estructura y funcionamiento de nuestros servicios de salud mental.

Discusiones sobre el significado concreto del término rehabilitación y sus diversos componentes, sobre si todos los profesionales de salud mental «hacen», deben o pueden «hacer» rehabilitación o ésta es una tarea reservada a personal más especializado, sobre la relación entre rehabilitación y apoyo social, sobre la necesidad o no de dispositivos específicos o sobre el carácter sanitario o no sanitario de distintas actividades, servicios y profesionales de la rehabilitación, son, entre otros muchos, algunos de los debates que de manera directa o indirecta se mantienen periódicamente entre nosotros ${ }^{1}$. El debate no sólo no es malo sino que, además de inevitable, puede resultar útil, ayudándonos al menos a definir mejor nuestro trabajo. Pero para ello es importante delimitar con precisión los términos del mismo y ahí nos encontramos muy a menudo con otro problema, el de que muchas de nuestras discrepancias se deben no

\footnotetext{
1 Por no hablar de «disquisiciones» más esotéricas, como si hay una rehabilitación «verdadera» y una «seudorehabilitación», tema abordado, por ejemplo, en una mesa redonda del Congreso Nacional «La salud mental es cosa de todos», organizado por el IMSERSO en Valencia en noviembre de 2004.
} 
sólo a diferencias conceptuales o de enfoque teórico, o a distintas consideraciones estratégicas o tácticas, sino también a distorsiones derivadas de confusiones e imprecisiones terminológicas.

Pero hay también dificultades prácticas que tienen relación con esas discrepancias, además de con otros muchos factores, entre ellos la evidente infradotación de recursos que suele caracterizar este sector. Se produce así no sólo una insuficiencia general de actividades, profesionales y espacios «rehabilitadores», sino también distorsiones curiosas, como la que luego llamaremos «paradoja de la rehabilitación», situación en la que la carencia de intervenciones «rehabilitadoras» en la atención a personas con trastornos mentales graves, coexiste con una escasa e inadecuada utilización de algunos recursos específicamente destinados a este fin, como son, por ejemplo en el caso de Andalucía, las unidades de Rehabilitación. Paradoja que algunos pretenden de vez en cuando solucionar, en un ejemplo del clásico «tirar por la calle de en medio», proponiendo, hasta el momento más o menos «en voz baja», la desaparición de dichas Unidades.

Esta situación no es específica de Andalucía, pero presenta aquí algunas características peculiares. Entre otras cosas por ser, pese a todo, nuestra Comunidad Autónoma una de las que más ha avanzado en el desarrollo de un sistema de atención comunitaria, tras la desaparición de los hospitales psiquiátricos públicos. Pensamos, por ello, que merece la pena reflexionar un poco sobre esta situación, proponiendo algunos elementos para un debate profesional que en nuestra opinión corresponde impulsar, entre otros agentes, a nuestra Asociación².

Como contribución a ese debate, y aunque en otros espacios hemos desarrollado de manera más extensa distintos aspectos de nuestra posición sobre el tema (3-10), vamos a intentar sintetizarlos, articulando algunos conceptos sobre atención comunitaria, rehabilitación y apoyo social. Pretendemos con ello ofrecer un esbozo de sistematización teórica, que pueda servir de base para una aproximación crítica a algunos aspectos de la situación concreta de Andalucía, en referencia a los distintos aspectos que incluimos bajo el término «rehabilitación». En ese sentido el artículo resume (quizás con más extensión y reproducciones textuales de las debidas) distintos planteamientos ya publicados (3-10), que intentan servir de hilo conductor para exponer, finalmente, algunas hipótesis valorativas sobre la situación actual y algunas estrategias que nos parece útil tener en cuenta para intentar mejorarla. Aunque nuestras reflexiones y propuestas se basan lógicamente en la situación de Andalucía, pensamos que, en sus aspectos más generales, pueden ser de interés para compañeros y compañeras de otras Comunidades Autónomas.

\footnotetext{
2 Aunque algunas de sus contribuciones (1-2) no estén exentas, en nuestra opinión y pese a su valor, de algunas confusiones del tipo de las aquí aludidas.
} 


\section{Un marco de referencia general. Atención comunitaria y personas con trastorno mental grave o severo}

Como hemos mencionado en otras ocasiones, hablar de atención comunitaria, rehabilitación y apoyo social en salud mental sigue significando todavía moverse en un espacio con importantes niveles de confusión e imprecisión (4; 5). El carácter polisémico de muchos de los términos que utilizamos, la complejidad de prácticas y posiciones teóricas y su desigual nivel de desarrollo tanto en general como, especialmente, en nuestro país $(1 ; 2 ; 11 ; 12)$, parecen ser algunos de los factores que determinan dicha confusión. Sin olvidar que, como señalaba hace unos años Benedeto Saraceno (13), estamos haciendo referencia a un campo que sigue caracterizándose, todavía, más como una «serie de prácticas en busca de teoría», que como un cuerpo teórico cerrado y coherente.

Por ello, para intentar ordenar el debate, aun a costa de dar un cierto «rodeo» argumental, vamos a exponer nuestra visión del tema, proponiendo algunas definiciones que faciliten una mayor precisión a la hora de establecer los términos de la discusión. Dado que una de las funciones ya tradicionales de la filosofía (14) (para algunos si no la única sí al menos la más relevante (15)) es la de ayudar a esclarecer los términos técnicos para aumentar su precisión y evitar debates estériles, por confusiones en su uso, empezaremos por una breve «excursión filosófica».

\subsection{La atención comunitaria como tecnología}

Desde esa perspectiva filosófica, una manera de situar el tema, a modo de marco general, es enfocar la Atención Comunitaria (y, dentro de ella, la «rehabilitación» y su prolongación en los programas de «apoyo social») como una «tecnología» $(4 ; 5)$, recurriendo a un término ya usual en la actual Filosofía de la Ciencia (14; 16-18). Con él se quiere hacer referencia a un complejo sistema de acciones sociales, diferenciado de otros como aquellos a los que denominamos «ciencia»o «artesanado», y que: articula conocimientos teóricos, procedimientos técnicos y agentes profesionales específicos (con sus expectativas, valores e intereses concretos, y no sólo como supuestos «entes racionales»); se basa en el desarrollo científico, en el doble sentido de ser, por un lado, compatible con los conocimientos científicos y técnicos disponibles en cada momento y, por otro de utilizar una metodología igualmente científica (19) («pensamiento lógico-racional» y algún tipo de evaluación o contrastación empírica); y cuya evolución no es entendible en función de factores exclusivamente internos, sino que debe ponerse en relación compleja con el desarrollo macro y micro social. 
La utilidad de aplicar esa triple dimensión (reflejada en la trilogía «ciencia, tecnología, sociedad» que da nombre a algunos enfoques actuales de la Filosofía de la Ciencia (16)), al análisis de un sistema como el nuestro, tiene que ver con algunas de sus implicaciones. De hecho, si definimos nuestro campo como una tecnología, estamos enfatizando la necesidad de una permanente revisión crítica de la consistencia de nuestras formulaciones teóricas y de su concordancia con los distintos conocimientos científicos relacionados con ellas, así como de la evaluación sistemática de nuestras técnicas de intervención. Lo que, entre otras cosas, enlaza con el «núcleo racional» de la llamada «atención basada en la evidencia» $(20 ; 21)$, si bien algunas de sus formulaciones, tanto generales como en salud mental, deben ser consideradas con una razonable «distancia crítica».

Pero esa definición implica también reconocer el papel central que corresponde, en el desarrollo de nuestra tecnología, a distintos agentes, profesionales y no profesionales. De ahí la importancia, además de la sistematización teórica y la investigación empírica, de la formación profesional y el debate teórico y técnico. Debate que, frente a lecturas mal informadas del término «tecnología», incluye inevitablemente referencias a «valores» (epistémicos, éticos, políticos y, en general, sociales), a filosofía y, en definitiva, a «ideología» (en el sentido más amplio del término) y no sólo a meras relaciones de técnicas y hechos concretos, «puros e incontaminados» $(14 ; 16 ; 17 ; 22)$.

Por todo ello y más allá de su interés filosófico general, pensamos que esta consideración, que tiene todavía más de deseo y referencia orientadora que de realidad plena, podría ayudarnos a superar progresivamente la persistencia de niveles importantes de error, desconocimiento y mala práctica que buscan refugio en el «artesanado» que, con mayor o menor fortuna, viene caracterizando gran parte de nuestra actividad profesional.

1.2. Un intento de definición «operativizable» de la atención comunitaria en salud mental

En ese contexto, lo que denominamos «Salud Mental Comunitaria»o «Atención Comunitaria en Salud Mental» debe verse, de manera un poco más precisa, como una determinada orientación - «paradigma tecnológico»o «programa de investigación tecnológico», si recurrimos a la extrapolación de dos términos también tradicionales en filosofía de la ciencia (23-25) - o enfoque teórico, organizativo y funcional, alternativo a otros modelos, como los que basan la atención en hospitales psiquiátricos, en servicios médicos más tradicionales («unidades o departamentos de Psiquiatría» de los hospitales universitarios, por ejemplo) o en 
sistemas tipo consulta individual médica o psicológica (4; 5). Atención Comunitaria que, pese a la diversidad de definiciones existentes (26-31), hemos caracterizado en otras ocasiones $(4 ; 5)$ a partir de los siguientes aspectos o dimensiones básicas:

a. Un planteamiento de «salud pública», basado en el compromiso de atención al conjunto de problemas de salud mental, de territorios y poblaciones concretas, así como en la utilización de criterios epidemiológicos tanto en el enfoque poblacional general como en el establecimiento de prioridades de atención.

b. Una filosofía de la rehabilitación ${ }^{3}$, que dirige las intervenciones preferentemente hacia el incremento de la autonomía personal y la funcionalidad social de usuarios y usuarias, enfatizando sus capacidades y respetando sus intereses y expectativas; lo que implica una atención integral, con actividades de prevención, tratamiento, rehabilitación (en sentido estricto) e integración social.

c. La organización de la atención mediante una red de dispositivos (sanitarios y no sanitarios), con capacidad para facilitar un conjunto amplio de prestaciones, accesibles y adaptables a las necesidades individuales; red que, además de sustituir y no meramente complementar los hospitales psiquiátricos, sitúa la mayoría de sus componentes fuera del ámbito hospitalario, lo más cerca e integradamente que sea posible de los «contextos de vida» de las personas a las que atiende.

d. Un funcionamiento que se basa tanto en el trabajo en equipo, con mecanismos de coordinación que aseguren la continuidad de cuidados, como en la participación de profesionales, usuarios y usuarias, familiares y otros grupos sociales.

En ese sentido, hablar de atención comunitaria significa, con respecto a la organización y funcionamiento de los servicios de salud mental, «algo» más que la mera desaparición formal de los hospitales psiquiátricos, con todo lo importante que resulta dicha desaparición (32). En términos organizativos implica contar con redes integradas de servicios, basados en equipos multiprofesionales y con una responsabilidad territorial clara. Y, en términos funcionales, basarse en criterios epidemiológicos, garantizar la continuidad de cuidados y favorecer en los y en las profesionales enfoques «rehabilitadores» en sentido amplio así como dinámicas participativas y de trabajo en equipo. Condiciones que, junto a una dotación suficiente de profesionales y dispositivos, con sus correspondientes estándares de 
calidad, pueden permitir funcionar establemente sin reconstruir espacios de exclusión ni dejar sin atender necesidades de usuarias, usuarios y familiares.

\subsection{El concepto de «trastorno mental grave» $\mathrm{o}$ «severo»}

Desde la perspectiva de una Atención Comunitaria así definida (enfoque poblacional y prioridades de atención) tiene sentido identificar grupos de población con tipos de problemas que requieren estrategias de intervención similares, más allá de la visión clínica individual y de la referencia diagnóstica específica. Uno de estos grupos es el que la literatura profesional internacional denomina «personas con trastorno mental grave o severo» $(1 ; 2 ; 4 ; 5 ; 6 ; 33$; $34)$, colectivo de referencia cuando hablamos de rehabilitación y apoyo social en salud mental. El término sustituye además al ya histórico «crónico», impreciso, desajustado y demasiado dependiente, al igual que su opuesto «agudo», del modelo de clasificación manicomial $(4 ; 5,35 ; 36)$, para hacer referencia a «personas con dificultades de funcionamiento personal y social, derivadas de padecer problemas de salud mental de carácter severo y persistente, en nuestras sociedades». Identificamos así un grupo de personas que requieren una atención preferente, dada la multiplicidad y gravedad de sus problemas, la existencia de intervenciones de efectividad razonable y su carácter crítico para la atención comunitaria, ya que éste era el núcleo fundamental del colectivo «atendido» en los hospitales psiquiátricos (32) y sus dificultades de manejo, desde modelos comunitarios, suelen servir de coartada a los defensores del sistema manicomial.

A pesar de una inicial heterogeneidad en su delimitación, parece haber un relativo consenso sobre las dimensiones que deben tenerse en cuenta para su definición operativa $(1 ; 2 ; 4 ; 5 ; 6 ; 33 ; 34 ; 37)$, incluyendo básicamente:

a. Una sintomatología que podríamos resumir en términos generales como «psicótica», en la medida en que genera dificultades de captación de la realidad y de manejo, entre otras cosas, de las relaciones interpersonales. Lo que traducido a diagnósticos, suele incluir fundamentalmente esquizofrenia y otras psicosis delirantes (grupo diagnóstico mayoritario), psicosis afectivas y algunos tipos de trastornos de personalidad (los más «cercanos» a las psicosis).

b. Una evolución prolongada en el tiempo (fijada habitualmente en más de dos años), lo que implica además una utilización continuada o muy frecuente de distintos tipos de recursos sanitarios y sociales, entre los que se hace hincapié en los de hospitalización. 
c. Un componente de discapacidad, que suele medirse con instrumentos estandarizados como el GAF (38) e implica la presencia de alteraciones en varios aspectos funcionales, como el alojamiento, la conducta social, el funcionamiento en la vida cotidiana, las relaciones sociales y el empleo.

Dimensiones a las que creemos que hay que añadir otra de carácter «contextual», referida a la existencia de un medio social próximo poco tolerante y/o «agotado», resaltando así una dimensión contextual o relacional, no siempre fácil de medir pero que no deberíamos perder de vista, ni a la hora de analizar la situación de las personas a las que debemos atender ni a la de intervenir para mejorarla.

\subsection{Algunos modelos referencia}

Para entender las características de estas personas hay también algunas perspectivas o «modelos» que pueden servir de referencia para establecer estrategias razonables de intervención, dentro de la Atención Comunitaria $(2 ; 5)$. Perspectivas y modelos que, en su mayor parte, se vinculan también a los distintos desarrollos teóricos y tecnológicos de ese amplio movimiento que solemos englobar bajo el término de «Rehabilitación Psicosocial» $(2 ; 13 ; 21 ; 39-45)$.

\subsubsection{De «loco» o «loca» a «ciudadano» $\mathrm{o}$ «ciudadana»}

Así, en el nivel más general, es útil hacer referencia a lo que podríamos denominar consideración social de las personas con este tipo de problemas, y específicamente a la evolución de la misma a lo largo de la segunda mitad del pasado siglo, en estrecha relación con distintos procesos de desinstitucionalización, con sus componentes profesionales y políticos $(4-6 ; 13 ; 27 ; 32 ; 46)$. Con fines didácticos hemos resumido en otras ocasiones $(4 ; 5)$, de manera un tanto simplista pero sugestiva, esa evolución a partir de la transición entre los términos «loco/a» (que requiere aislamiento y reclusión por su supuesta peligrosidad), «enfermo/a» (susceptible por tanto de atención sanitaria) y «ciudadano/a» (13). Salto, este último, que implica una perspectiva más amplia, aunque por el momento aún más teórica que real, que enfatiza su consideración como personas con derechos y deberes, para cuyo ejercicio encuentran dificultades múltiples, derivadas tanto de su enfermedad como de sus consecuencias en términos de discapacidad personal, pero también de la existencia de barreras sociales, ligadas al estigma.

Consideración que, por otra parte, nos sitúa de lleno en la perspectiva de la atención comunitaria tal y como la hemos definido anteriormente, al resaltar la 
necesidad de un conjunto variado de intervenciones y competencias, así como la implicación de distintos sectores administrativos y profesionales, de movimientos asociativos de las personas más directamente afectadas (usuarias y familiares) y de distintos agentes de la llamada sociedad civil.

\subsubsection{Los modelos de «vulnerabilidad»}

Un segundo aspecto a considerar es el referido a la articulación de los distintos factores biopsicosociales que parecen tener algún tipo de relevancia en la aparición, desarrollo y posibilidades de intervención en este tipo de problemas. Y ahí, al menos en relación con el grupo mayoritario, constituido por los que englobamos bajo el término genérico de «esquizofrenia», hay que hacer referencia a los modelos operativos de «vulnerabilidad» $(2 ; 39 ; 47-49)$, que facilitan un razonable «armazón conceptual», útil para orientar tanto la investigación como la organización de programas individualizados de intervención. Así, la diferenciación, por un lado, entre condiciones de vulnerabilidad, episodios clínicos y formas evolutivas a medio y largo plazo (48) y, por otro, el juego interactivo entre distintos factores biológicos (48) -que parecen tener repercusiones cognitivas comunes $(50 ; 51)$-, precipitantes psicosociales del tipo de los acontecimientos vitales o los niveles de emoción expresada $(39 ; 52)$, y factores protectores (habilidades de manejo y redes sociales), indican tanto líneas de investigación como de intervención de efectividad razonable (53), a las que haremos referencia más adelante.

\subsubsection{Discapacidad y trastorno mental grave}

Otro aspecto importante es el referido a la aplicación en este campo del grupo de conceptos relacionados con las dimensiones de «discapacidad» («deterioro», «discapacidad», «minusvalía»), elaborados en día por la OMS, como base para entender un grupo más amplio de problemas, superando las visiones más tradicionalmente médicas y enlazando con aspectos funcionales, que de nuevo incluyen elementos contextuales de carácter psicológico y social $(2 ; 42)$. Su reciente revisión introduce enfoques y terminología novedosa $(2 ; 54 ; 55)$, que contribuye a una mejor caracterización de los efectos de la discapacidad, más adaptada también a las características de esta población (56), a la vez que elimina algunos errores e insuficiencias del enfoque anterior. Básicamente los cambios afectan al enfoque teórico (perspectiva social y universal frente al predominio biomédico), a la visión global (aspectos positivos «funcionamiento» y no sólo negativos «discapacidad», con inclusión además de factores contextuales) y a lo que todo ello implica sobre la terminología: «discapacidad» como termino global que incluye dimensiones 
relacionadas con los «cambios en las estructuras y funciones corporales», las «limitaciones en la actividad» y las «restricciones en la participación», sin postular relaciones de causalidad lineal entre unas y otras, como sugería el modelo anterior.

Conceptos generales y clasificaciones concretas que se adaptan razonablemente a las características de las personas con trastornos mentales graves (56), introduciendo nuevas dimensiones en la valoración de sus problemas, siempre en la línea de resituar el papel del enfoque y la intervención sanitaria, que deben articularse en el marco de una visión mucho más amplia, tanto de los problemas como de las posibilidades de intervención.

\subsubsection{El concepto de «recuperación»}

Y por último habría que mencionar también el concepto más reciente de «recuperación» («recovery») (57-59), desarrollado a partir de la confluencia de diferentes perspectivas del movimiento asociativo de usuarios y usuarias $(59 ; 60)$ y de estudios epidemiológicos de seguimiento a largo plazo de personas con graves problemas de salud mental $(61 ; 62)$. En conjunto, tanto la visión de las personas asociadas, como los datos derivados en los estudios epidemiológicos (y que muestran más del 50\% de evoluciones razonables a largo plazo) van facilitando una imagen muy diferente de la tradicional, tanto en lo relativo a sus posibilidades de recuperación personal y social, cuanto al protagonismo de las personas directamente afectadas, cuyo papel activo intenta acentuar el no fácilmente traducible término «empowerment» (63). Imagen que va integrándose progresivamente en la reorganización de servicios y programas en distintos modelos de atención comunitaria (64), a la vez que da pie a debates importantes $(63 ; 65 ; 66)$ en los que no podemos entrar aquít.

\section{Hacia una visión integradora}

En conjunto estas aproximaciones contribuyen a establecer un campo teórico específico para el entendimiento de los problemas de las personas con trastorno mental grave, bastante diferente de lo que podemos denominar «visiones clínicas tradicionales». Al respecto caben también dos perspectivas alternativas

4 Como en muchas otras ocasiones, algunas perspectivas extremas, que enlazan en cierta medida con posiciones derivadas de la llamada «antipsiquiatría», son tan discutibles como lo son, en sentido opuesto, las derivadas de los enfoques parciales (restrictivamente «biológicos» o restrictivamente «psicológicos») de algunas de nuestras «escuelas» habituales. 
(63; 66-68), según consideremos que debemos verlas básicamente como personas afectadas por una enfermedad y necesitadas por tanto de intervenciones sanitarias, que, a veces, hay que complementar con distintos tipos de actuaciones y apoyos sociales. O, desde otro punto de vista, como miembros de uno de los colectivos atrapados en condiciones sociales de discriminación o exclusión, que requieren, en una sociedad democrática, distintos tipos de intervenciones sociales (en sentido amplio), entre las que figuran evidentemente algunas de carácter sanitario ${ }^{5}$.

De modo más pragmático y, en nuestra opinión más útil, cabe integrar ambas visiones en un enfoque realmente biopsicosocial, que, en la perspectiva de la atención comunitaria, exige la articulación de intervenciones múltiples, de naturaleza intersectorial, en las que, con un objetivo final de inclusión social y recuperación de la ciudadanía, hay un espacio común tanto para las intervenciones sanitarias como para las no sanitarias $(4-8 ; 13 ; 69)$. Enfoque que, con sus correspondientes implicaciones para la rehabilitación y el apoyo social, pone el acento en:

a. La insuficiencia e inadecuación de una visión exclusiva o preponderantemente sanitaria, a la que se contraponen perspectivas ciudadanas y sociales más amplias.

b. Las diferentes dimensiones relevantes en este tipo de problemas, con énfasis creciente, más allá de las puramente «clínicas», en las funcionales y de calidad de vida, que enlazan además con un importante componente subjetivo (derechos, expectativas, objetivos, participación).

c. Y las posibilidades reales de recuperación total o parcial y no sólo de cuidado meramente paliativo, que cabe considerar en una parte importante de los casos.

Aspectos que están en la base de las políticas y programas de rehabilitación y apoyo social en la atención comunitaria a las personas con este tipo de problemas, al definir no sólo los objetivos generales de la intervención pública (recuperación del funcionamiento personal y social, así como de la ciudadanía), sino también el alcance de las distintas intervenciones concretas (educar o reeducar para la vida social y ayudar para ello a construir y mantener apoyos, coherentes, sostenidos y respetuosos).

5 Si se hacen desde un enfoque que subordina el control de síntomas y/o conductas molestas o disruptivas a objetivos de recuperación e inclusión social y no a la inversa. 


\section{La organización de la atención a personas con trastorno mental grave en sistemas comunitarios}

Desde esa perspectiva general, y en contextos sociales como el nuestro, corresponde a los sistemas públicos establecer las intervenciones que pueden resultar efectivas para mejorar distintos aspectos de la situación de las personas con trastornos mentales severos y organizar los sistemas de atención para facilitárselas, basándose para ello en la información científica y tecnológica disponible y adecuándose a las características concretas de cada país. Si bien el primer aspecto, el de las intervenciones, registra un consenso creciente, el relativo a la estructura y organización de los recursos sanitarios y sociales que permiten aplicarlas es mucho más diverso, al ser estos aspectos bastante más dependientes de los contextos sociales (culturales, políticos, administrativos) concretos en que se desarrollan.

\subsection{Un conjunto de intervenciones «de efectividad razonable»}

Con respecto a las técnicas de intervención, es necesario por tanto tener en cuenta la información acumulada sobre su eficacia y efectividad. De hecho, a pesar de las discrepancias en las posiciones teóricas y de las aún mayores disparidades en las prácticas reales, se ha ido generando, en las últimas décadas, un creciente consenso técnico, con respecto a una serie de intervenciones que cuentan, al menos, con estudios publicados de suficiente rigor metodológico (con los estándares habituales en este campo) como para afirmar que pueden resultar de utilidad para las personas con este tipo de problemas $(4-6 ; 20 ; 21)^{6}$.

Ese consenso profesional señala la necesidad de un conjunto amplio, cuya composición, intensidad y duración, debe adaptarse a las variables necesidades individuales de los pacientes $(2 ; 4-6 ; 39 ; 70-76)$. En conjunto se incluyen tanto intervenciones directas sobre la persona afectada, como sobre su entorno inmediato (habitualmente la familia) y sobre distintos niveles y estructuras sociales, y, además de los fármacos, forman parte de este repertorio básico, un número importante de técnicas psicosociales, gran parte de las cuales se han desarrollado en gran medida dentro del campo de la llamada «rehabilitación psicosocial» $(2 ; 21 ; 34 ; 39$ 45;77-79).

\footnotetext{
6 La mayor parte de la bibliografía que citamos se centra en las personas con diagnóstico de esquizofrenia, tipo de trastorno (o trastornos) más común y de abordaje más complejo dentro del grupo de trastornos mentales graves o severos.
} 
El cuadro 1 ofrece lo que, en nuestra opinión, puede ser una sistematización razonable, teniendo en cuenta que se trata más de un inventario de «mínimos» que de «máximos». Como puede verse en él, con excepción de la medicación, el apoyo personal más o menos sistematizado, las técnicas psicoterapéuticas de base cognitiva y las actuaciones sobre el estigma, se trata efectivamente de intervenciones incluidas en la «oferta» habitual de los profesionales especializados en rehabilitación. Lo cual no significa, como luego insistiremos, que sólo puedan ser aplicadas por dispositivos y personal superespecializados, sino que deberían, por el contrario, formar parte del inventario básico de conocimientos y competencias, al nivel pertinente en cada caso, del conjunto de profesionales y dispositivos de una red comunitaria de Salud Mental (5).

Cuadro 1

Intervenciones sobre personas con trastorno mental severo

\begin{tabular}{|c|}
\hline Intervenciones directas con la persona con trastorno mental severo \\
Fármacos $(80)$ \\
Apoyo personal continuado $(71 ; 81-85)$ \\
Terapia cognitiva $(84-88)$ \\
Psicoeducación $(52 ; 84 ; 85 ; 89 ; 90)$ \\
Recuperación y/o desarrollo de habilidades personales $(39 ; 84 ; 85 ; 91-93)$ \\
Rehabilitación cognitiva $(84 ; 85 ; 93-96)$ \\
\hline Intervenciones con la familia y/o entorno social próximo \\
Psicoeducación familiar $(84 ; 85 ; 88 ; 97 ; 98)$ \\
\hline Provisión de recursos «instrumentales» \\
Alojamientos $(7 ; 99-102)$ \\
Actividad productiva y empleo $(8 ; 99 ; 103-105)$ \\
Interacción social y autoayuda $(52 ; 60 ; 63)$ \\
Dinero $(99)$ \\
Defensa jurídica y tutela $(99)$
\end{tabular}

2.2. Redes de servicios, equipos profesionales y estructuras de gestión

La prestación personalizada y continuada en el tiempo de distintas combinaciones de este conjunto de intervenciones, exige redes complejas y bien coordinadas de dispositivos y equipos profesionales $(4-6 ; 26 ; 31 ; 71 ; 99 ; 108)$ que, en nuestras sociedades, suelen tener un componente sanitario y otro u otros que podemos denominar «social»o «sociales» (en sentido amplio, no estrictamente superponible con el campo específico de los Servicios sociales), aunque esta división sea muy variable según los distintos contextos administrativos. 


\subsubsection{Dispositivos sanitarios}

Los servicios sanitarios especializados en salud mental (cuadro 2) suelen articularse sobre una base territorial, en torno a Centros, Unidades o Equipos Comunitarios de Salud Mental $(31 ; 109 ; 110)$ que enlazan, por una parte con los servicios sanitarios generales -especialmente con el nivel de Atención Primaria (111)- y, por otra, con un conjunto de dispositivos de mayor especificidad, entre los que suelen ser habituales los de hospitalización -en hospitales generales pero también en espacios no sanitarios $(31 ; 112-114)-$, a su vez diferenciados (31) en función de la duración (Unidades de corta estancia, Unidades de media y larga estancia de carácter activo), o cobertura horaria (hospitales y programas de día).

Son también frecuentes dispositivos específicos de rehabilitación (Centros de Día o Unidades de Rehabilitación $(1 ; 2 ; 31 ; 44 ; 108)$ ), y cada vez más se desarrollan dispositivos, programas y / o equipos específicos $(31,109,110)$ para determinados tipos de problemas o situaciones relacionados preferentemente con personas con trastorno mental grave: atención a urgencias/ intervención en crisis, programas de gestión de casos y/o de seguimiento intensivo $(31 ; 116)$, intervención precoz, etc.

\section{Cuadro 2}

Dispositivos y programas sanitarios para la atención a personas con trastorno mental severo $(4 ; 5 ; 31 ; 108 ; 109 ; 110 ; 115)$

\begin{tabular}{|c|}
\hline Atención Primaria \\
Equipos / Centros de Salud \\
Equipos de Enfermería comunitaria \\
\hline Servicios generales de Salud Mental \\
Centros / Equipos de Salud Mental Comunitaria \\
\hline Unidades de Hospitalización \\
Unidades de corta estancia en Hospitales Generales \\
Unidades / Equipos de hospitalización alternativa \\
Unidades de Media-larga estancia \\
Hospitales de día \\
Programas de Hospitalización parcial \\
\hline Unidades de rehabilitación \\
Centros de día /Unidades de rehabilitación \\
\hline Dispositivos y programas específicos \\
Equipos de intervención en crisis \\
Programas de gestión de casos \\
Equipos de Tratamiento Asertivo Comunitario \\
Equipos de intervención precoz
\end{tabular}




\subsubsection{Dispositivos de apoyo social}

Pero, además de los dispositivos descritos, habitualmente incluidos en el sector sanitario, hay un conjunto de servicios e intervenciones que, habitualmente y en nuestras sociedades, se sitúan administrativamente fuera del mismo, aunque de nuevo aquí nos encontramos con alguna controversia de interés.

De lo que estamos hablando básicamente es de la provisión de lo que hemos llamado «recursos instrumentales», es decir de facilitar, a las personas con dificultades derivadas y/o «añadidas» a su enfermedad mental, distintos elementos de apoyo que tratan de contrarrestar sus dificultades y problemas y a eliminar algunas de las barreras que bloquean su pleno funcionamiento ciudadano (4; 69; 99). Y para ello es pertinente la discusión sobre si son necesarios recursos de apoyo social, específicos para personas con trastorno mental severo, o bastaría con utilizar los habitualmente disponibles en el campo de los servicios sociales ${ }^{7}$, educativos, laborales, etc. De modo similar a la estrategia sanitaria de la atención primaria de salud, la insistencia en utilizar recursos generales de Servicios Sociales, Educación, Trabajo o Cultura, no implica en nuestra opinión negar la necesidad de programas, dispositivos y profesionales especializados, dentro de estos sectores de intervención, para personas con dificultades tan específicas como aquellas con las que trabajamos. Consideración que, más allá de las posiciones o «declaraciones de principio», más o menos ingenuas de algunos, forma parte de cualquier planteamiento serio de la atención comunitaria en distintos lugares del mundo, situándose las diferencias en la tipología, dotación y dependencias administrativas de dichos recursos $(4 ; 31 ; 44 ; 69 ; 71 ; 76 ; 99$; $115)$, que muestran una gran variabilidad.

Aunque no siempre resulta fácil precisar dónde termina lo específicamente sanitario y empieza lo «social», podemos guiarnos por los criterios utilizados, desde sectores no sanitarios, para otros colectivos con dificultades de funcionamiento social (políticas de alojamiento, empleo, pensiones y subsidios, educación, etc.). Con esa referencia consideramos que es necesario incluir en este apartado el tipo de programas que resume el cuadro 3.

Que, por su parte diferencian habitualmente entre unos de carácter «general», es decir, comunes a todo tipo de personas con carencias sociales, y otros «especializados», es decir, específicos para determinados colectivos (personas mayores, personas con deficiencia mental, etc.), que no suelen incluir a personas con enfermedad mental por el efecto histórico de los hospitales psiquiátricos, considerados durante muchos años como el «espacio natural» de los enfermos y enfermas mentales. 
Cuadro 3

Programas de apoyo social específicos para personas con trastorno mental severo

Red de alojamientos y programas residenciales $(7 ; 44 ; 99-102)$
Rehabilitación vocacional y programas de empleo $(8 ; 44 ; 99 ; 103-105)$
Programas y dispositivos ocupacionales $(8 ; 44 ; 31 ; 52 ; 99)$
Programas de recuperación educativa $(92)$
Actividades de ocio y empleo del tiempo libre $(31 ; 44 ; 52 ; 99)$
Actividad asociativa $(52 ; 60 ; 63 ; 99)$
Ingresos económicos y apoyo en su gestión $(99)$
Mecanismos para la tutela $(99)$

Dispositivos y programas que no substituyen sino que complementan el acceso a las prestaciones habituales de los distintos sistemas de servicios públicos (servicios sociales generales, sistema educativo, mecanismos de protección social, políticas de empleo, etc.), a las que, como el resto de los ciudadanos y las ciudadanas, con y sin discapacidad, tengan derecho.

\subsubsection{Equipos multiprofesionales}

A diferencia de los sistemas tradicionales y de otros modelos sanitarios, la atención comunitaria en salud mental implica una atención compleja por parte de equipos profesionales de composición múltiple y dinámica interactiva (4-6; 31; $109 ; 110 ; 115)$. La multiplicidad de áreas a cubrir y la complejidad de competencias profesionales requeridas para ello rompen con la imagen tradicional del Hospital Psiquiátrico (personal médico en un extremo, auxiliares en otro y en medio poco más) y la de otras especialidades sanitarias (donde aumenta la presencia del personal de enfermería, con una intervención cada vez más rigurosa pero también más independiente). Así la atención comunitaria implica personas de muchas y variadas profesiones, tanto sanitarias (psiquiatría, psicología clínica, enfermería, trabajo social, terapia ocupacional, etc.) como no sanitarias. Aunque, en este último caso, muchas de ellas están todavía lejos de tener una clara definición profesional y una titulación reconocida (monitores residenciales y ocupacionales, orientadores laborales, técnicos y técnicas de apoyo al empleo, personal de empresas sociales, animadores y animadoras socioculturales, etc.).

Por otro lado y enlazando con lo que decíamos en el apartado referido a los dispositivos, los sistemas comunitarios de carácter público hacen énfasis en equipos genéricos $(31,109,117)$, polivalentes y bien distribuidos territorialmente, como lugar de enlace con la atención primaria, por un lado, y con equipos específicos para la hospitalización y la rehabilitación especializada, por otro. Pero cada vez más esta estructura se completa con equipos más especializados, inicialmente desarrollados en el marco de sistemas privados o mixtos (en 
gran parte en EE.UU.), pero que han demostrado ser útiles para determinados tipos de problemas, momentos o situaciones: atención en crisis, atención en primeros estadios, tratamiento asertivo, etc. Aunque el encaje diste de ser siempre perfecto, el futuro parece estar en esa articulación entre equipos genéricos y especializados, intentando garantizar en cualquier caso la llamada «continuidad de cuidados» (109).

Por último, en todos ellos el modelo de atención comunitaria que defendemos aquí, sin renunciar al desarrollo competencial propio de cada profesional, pone el énfasis en modelos de cooperación («trabajo en equipo») basados en relaciones transversales más que jerárquicas, en la flexibilidad de roles y en el aprovechamiento local de las diferentes capacidades de cada miembro del equipo (109). Funcionamiento que no siempre se ve facilitado por las aparentemente inevitables tensiones corporativas, agravadas por los modelos imperantes en otros sectores de la atención sanitaria que, cuando hablan de trabajo en equipo, suelen entenderlo más como la mera coexistencia de distintos profesionales y la articulación secuencial de su trabajo (tipo «cadena de montaje»).

\subsubsection{Dependencias administrativas y mecanismos de gestión}

En general y en lo que respecta a los aspectos de dependencia administrativa y de gestión, la diversidad es mucho mayor, tanto en lo referido al sistema sanitario como, especialmente, a los recursos de apoyo social.

En términos generales y a diferencia de la postura «ecléctica» adoptada por la organización Mundial de la Salud $(115 ; 118)$, nosotros defendemos un modelo de responsabilidad y gestión pública, integrado siempre que sea posible dentro de los sistemas generales de servicios sanitarios y sociales, como el más adecuado a los principios de la atención comunitaria. Y ello no sólo por «ideología» (en definitiva, en este caso, defensa del valor de la responsabilidad colectiva en la cobertura de necesidades sociales muy sensibles a la inequidad) sino por un análisis razonable (aunque evidentemente discutible) de la dificultad de organizar y hacer funcionar servicios tan complejos como los aquí referidos sobre la base del «mercado» y una paralela desconfianza sobre el interés y capacidad del sector «privado» para asegurar estos servicios en sociedades como la nuestra. Aunque la «evidencia» no es concluyente, creemos que hay elementos suficientes en la experiencia internacional como para sustentar esta opinión, aunque no podamos extendernos aquí en el tema.

En lo que respecta al sistema sanitario, el planteamiento actual en nuestro país coincide al menos formalmente con nuestra visión, aunque la adecuación «a» y «de» los mecanismos de gestión habituales del sistema sanitario deje todavía mucho que desear, al igual que otros aspectos de la reforma psiquiátrica (119). 
$\mathrm{Y}$ en lo que respecta a los dispositivos y programas no sanitarios, la experiencia internacional parece indicar que es posible una mayor variabilidad en la responsabilidad administrativa y la gestión $(1 ; 4 ; 31 ; 44 ; 69 ; 71 ; 99,108 ; 115$; $119 ; 120)$. Siempre desde la perspectiva de la responsabilidad pública, es posible una adscripción administrativa unitaria o múltiple, situando, por un lado, cada tipo de servicio concreto en las correspondientes administraciones de Servicios Sociales, Empleo o Educación, y, por otro, poniendo el acento en la organización local o regional. Y, en el primer caso, como podemos ver en distintos ejemplos de nuestro país, cabe adscribirlos a una administración concreta (Servicios Sociales en el caso de Madrid) o una estructura intersectorial específica (FAISEM en Andalucía, FISLEM en Castilla La Mancha, etc.). Esta última solución $(4 ; 5 ; 69 ; 121 ; 122)$ nos parece que tiene ventajas claras y puede ser una de las principales opciones a defender en conexión con el futuro sistema nacional de Dependencia.

\section{Atención comunitaria, rehabilitación y apoyo social}

Como conclusión provisional de todo lo anterior podemos intentar ahora articular mejor las relaciones entre rehabilitación, atención comunitaria y recursos de apoyo social, proponiendo algunos temas que permiten, en nuestra opinión una mejor ordenación del necesario debate (o debates) sobre el tema, así como formulando, desde ellos, algunas preguntas que nos parecen adecuadas como guía para la evaluación de nuestros sistemas de atención comunitaria. Pero antes nos tenemos que enfrentar directamente con el término «rehabilitación», cuyo carácter polisémico hemos pretendido subrayar a lo largo del texto mediante el empleo de comillas.

\subsection{El término «Rehabilitación» y su(s) uso(s) en Salud Mental}

Como hemos propuesto también en otras ocasiones $(4 ; 5)$, el término requiere un análisis previo, dada la multiplicidad de sentidos con que se usa y las confusiones que ello suele acarrear. Para ello recurrimos de nuevo a un esquema, aplicado hace unos años al término «Atención Primaria de Salud»", que nos parece útil para ordenar esa multiplicidad de sentidos, diferenciando en

\footnotetext{
8 Diferenciando, en este caso, una filosofía, una estrategia, un nivel de cuidados y un conjunto de prestaciones concretas (123).
} 
este caso cinco tipos o niveles de uso, evidentemente interrelacionados, pero que introducen matices importantes en relación con algunos temas centrales de la atención comunitaria:

1. Una filosofía general (34), que integra un enfoque global de los problemas de las personas con trastornos mentales severos, junto al correspondiente planteamiento de los objetivos de su atención. Así, dichos problemas se consideran en términos de «desventaja social» (dificultades concretas que cada persona encuentra para manejarse en su medio habitual) y no sólo de «enfermedad» y se orientan las intervenciones no necesariamente hacia la «curación» (signifique esto lo que signifique, desde el mero control de síntomas hasta una ideal «reestructuración intrapsíquica»), sino hacia la consecución de la mayor capacidad posible para desenvolverse en su contexto social con el mínimo de dificultades. Objetivo al que evidentemente también pueden contribuir las «curaciones» 0 «mejorías clínicas».

2. Una estrategia (124), que pone el énfasis, como en el apartado anterior, en el trabajo con las capacidades-discapacidades de las personas (en definitiva con sus distintas habilidades y limitaciones y no con los síntomas, las lesiones anatomopatológicas o las dinámicas inconscientes), así como en su protagonismo y participación activa «empowerment» (63); estrategia que parece especialmente adecuada para las personas con trastornos mentales severos. Tanto en este sentido como en el anterior, la Rehabilitación englobaría en la práctica el conjunto de las intervenciones sanitarias y sociales, siempre que partiesen de un enfoque global, que considera a la persona con su historia, intereses, expectativas, capacidades y problemas concretos, a la vez que inserta en el contexto micro y macrosocial en que se desenvuelve.

3. Un nivel de intervención, que en el clásico esquema de la Salud Pública $(125 ; 126)$, se sitúa junto a la promoción de la salud, la prevención de la enfermedad, el tratamiento y la reinserción social; aunque en nuestro campo esas distinciones no sean tan fáciles de establecer y presenten en la práctica fronteras más bien difusas.

4. Un conjunto de programas, cuya utilidad para mejorar la situación de dichas personas, cuenta con algún grado de «evidencia» empírica $(2 ; 21$; $40 ; 77 ; 79)$, y que forman parte del inventario de procedimientos de la llamada rehabilitación psicosocial. Se incluyen aquí programas de rehabilitación cognitiva, entrenamiento en habilidades sociales, desarrollo de habilidades de la vida diaria, psicoeducación personal y familiar y reha- 
bilitación vocacional, pudiendo ordenarse según niveles de complejidad, especialización y hasta, si se quiere, de dependencia administrativa (sanitaria y no sanitaria, por ejemplo).

5. Y, finalmente, para acabar de complicar las cosas, un determinado tipo de dispositivos (en nuestro caso las unidades de rehabilitación). Dispositivos que varían en nombre y funcionamiento según diferentes contextos y combinaciones (mismo nombre y diferente función, misma función y diferente nombre, etc.), pero que no permiten afirmar, a priori, ni que todo lo que hacen es rehabilitación, ni que sólo se puede considerar rehabilitación lo que ellos hacen.

Es fácil constatar que según utilicemos el término «rehabilitación» con uno u otro de dichos sentidos, cambian las relaciones entre atención comunitaria, rehabilitación y apoyo social, o se entienden de distinta manera aspectos como la relación entre intervenciones y servicios. Lo que explica las dificultades de debatir ordenadamente sobre estos temas sin delimitar claramente a que nos estamos refiriendo en cada caso cuando hablamos de «rehabilitación».

\subsection{Relaciones entre atención comunitaria, rehabilitación y apoyo social}

Desde nuestra perspectiva, por tanto, y en relación con la atención a personas con trastornos mentales graves en sistemas basados en el paradigma de atención comunitaria en salud mental, no cabe hablar entonces de «la» rehabilitación en términos globales, ni a efectos de discutir su papel y/o ubicación, en general, dentro del conjunto de intervenciones y servicios, ni a la de valorar esos aspectos en sistemas concretos. Si lo que venimos planteando es correcto, los debates teóricos y los análisis prácticos deben tener en cuenta los distintos niveles de uso del término, que hacen referencia a aspectos muy diversos, aunque evidentemente también interrelacionados.

1. En el nivel global de la Atención comunitaria y considerando que ésta se organiza en redes de servicios y equipos profesionales tanto sanitarios como no sanitarios, es pertinente hablar del papel de la rehabilitación en un sentido amplio, entendiéndola como filosofía y estrategia. En ese sentido, para nosotros la rehabilitación es una característica básica definitoria del conjunto de la atención comunitaria y no de un determinado tipo de dispositivos, equipos o profesionales, ni de un determinado sector o adscripción administrativa (sanitario, social, laboral, educativo, etc.). Lo 
que significa (5) que todos y cada uno de los dispositivos y profesionales, que configuran una red comunitaria de salud mental, deberían basar sus intervenciones en consideraciones rehabilitadoras, en el sentido de orientarlas hacia la consecución del mayor nivel posible de funcionalidad personal y social de cada una de las personas atendidas, a la vez que deberían incorporar realmente, en todos los niveles de la red, la sensibilidad general sobre las dimensiones funcionales asociadas a la enfermedad, la consideración de cada usuario o usuaria como protagonista del proceso, y la consiguiente inclusión de estos aspectos en la evaluación de necesidades $(127 ; 128)$ y en la programación y desarrollo de las intervenciones concretas (2).

2. En lo que respecta al sistema sanitario son pertinentes además dos tipos de consideraciones sobre rehabilitación (5), utilizando ahora el término en sentido más restringido (programas de intervención y dispositivos y equipos profesionales):

a. Si hablamos de programas de rehabilitación psicosocial, hay que facilitar desde los servicios sanitarios aquellas técnicas sobre cuya efectividad hay un razonable consenso profesional: rehabilitación cognitiva, recuperación o desarrollo de habilidades personales y sociales, y psicoeducación de los pacientes y de sus familiares. Grupo de intervenciones que, con distintos grados de estructuración, intensidad y especialización, debe ser por tanto un componente básico de la atención sanitaria, como recoge por otro lado en nuestro país, aunque sea en una formulación muy general, el Decreto sobre prestaciones básicas del Sistema Nacional de Salud.

b. Y, si hablamos de dispositivos y profesionales, un sistema de atención comunitaria de calidad necesita disponer de profesionales con capacidad para aplicar esos programas concretos de rehabilitación (recuperación y/o desarrollo de habilidades concretas de funcionamiento personal y social) en muy distintos lugares de su red (básicamente en todos los que mantienen una relación prolongada con las personas con trastorno mental grave). Pero también parece razonable disponer en ella de algún dispositivo y/o equipo profesional específicamente capacitado, tanto para la evaluación funcional como para la aplicación de programas especializados y complejos de rehabilitación. Siempre que entendamos su función no sólo como aplicación directa de los mismos, en los casos de mayor complejidad y dificultad, sino también y quizás fundamentalmente, como apoyo al desarrollo de este tipo de programas en el conjunto de la red (incluyendo 
la investigación y la formación de personal). Dispositivos y profesionales que, en nuestra opinión, deben tener una adscripción sanitaria, reforzando así la dimensión psicosocial de los servicios de salud mental y facilitando la formación, en la misma, del conjunto de los profesionales.

3. En lo que respecta a los programas y dispositivos de apoyo social, habría que considerarlos como un elemento diferenciado de atención, que, aunque surgido en gran medida como prolongación de la atención sanitaria (desde el «tronco común» del hospital psiquiátrico), forma parte cada vez más de las políticas de inclusión social, dirigidas a favorecer el ejercicio del derecho de ciudadanía por parte de personas y colectivos con diferentes tipos de discapacidad. Enfoque que se enfatiza, por ejemplo, de modo creciente tanto en los programas residenciales $(7 ; 101 ; 129)$ como en los de empleo $(8 ; 103 ; 130 ; 131)$.

En su caso y en relación con la rehabilitación habría que repetir aquí algo de lo dicho anteriormente sobre la red sanitaria. En efecto, como parte de una red comunitaria más amplia (sanitaria y social), los dispositivos y profesionales de apoyo social se incluyen plenamente en el campo de la «rehabilitación», en sentido amplio (filosofía y estrategia, en nuestro esquema). Y, al mismo tiempo, suponen o deberían suponer en la mayoría de los casos, espacios y recursos personales privilegiados para el desarrollo de actividades de «rehabilitación» (en el sentido más restringido de «actividades dirigidas a la recuperación y/o desarrollo de habilidades concretas para el funcionamiento personal y social»), de distinto nivel de especialización. Aunque en todo caso dichas actividades deben realizarse con distintos grados de coordinación con el personal sanitario, cabe que algunas de ellas, de bajo nivel de complejidad, puedan ser desarrolladas por personal de la propia red de apoyo social.

4. Pero además, para que todo esto pueda funcionar como una red de atención comunitaria, es preciso definir explícitamente tanto el marco general de intervención, desde la perspectiva global del sistema de atención y para todos sus dispositivos y equipos profesionales («Programa de atención a personas con trastorno mental grave»), cuanto su concreción individual para cada persona a atender ( Programa individualizado de atención»). Lo que significa también establecer mecanismos de evaluación de necesidades y resultados, de formación y apoyo al personal, y los correspondientes espacios de coordinación, desde la discusión de casos a la gestión de los dispositivos y programas. 
3. 3. Algunas preguntas relevantes para la evaluación de la atención comunitaria

Situados en ese marco, hay también una serie de preguntas que pueden servirnos de guía en la evaluación de sistemas concretos de atención que dicen basarse en modelos comunitarios. Proponemos a continuación algunas de las más generales e intentaremos posteriormente tenerlas en cuenta para valorar, aunque sea de manera provisional y rudimentaria, algunos aspectos de la situación de Andalucía.

1. ¿Se basa la estructura y funcionamiento general de los servicios y equipos profesionales en un modelo comunitario, que hace énfasis en una filosofía de la rehabilitación?

2. ¿La actuación de los servicios y profesionales, en relación con las personas concretas objeto de atención, utiliza una estrategia de rehabilitación?

3. ¿Cómo se diferencian las intervenciones sanitarias y no sanitarias en áreas funcionales como la vivienda, la ocupación, el empleo o las relaciones sociales? ¿Dónde se pone la «frontera» en términos de organización y cómo se establece la coordinación en términos funcionales?

4. ¿Se aplican las intervenciones específicas de rehabilitación basadas en la evidencia en los casos y momentos adecuados y en qué lugares de la red?

5. ¿Hay unidades o equipos específicos de rehabilitación? Y, si es así ¿qué funciones cumplen y dónde se sitúan dentro de la red?

6. ¿Incluyen los sistemas de información indicadores periódicos de estructura, proceso y resultados que permitan conocer la evolución de estos aspectos y sus efectos sobre las personas atendidas?

4. Algunas implicaciones para el análisis de la atención comunitaria, la rehabilitación y la provisión de mecanismos de apoyo social en Andalucía

La exposición anterior tiene sentido en la medida en que pueda servirnos de ayuda, a la hora de situar los términos del debate en territorios y servicios concretos, es decir, en nuestro caso en Andalucía. Debate referido a la identificación de problemas, factores implicados y alternativas en relación con la atención comunitaria, la rehabilitación y los recursos de apoyo social a personas con trastorno mental grave.

Dado que no estamos en condiciones de abordar aquí un análisis riguroso de situación, con la información precisa y fiable que sería necesaria, nuestra pretensión es mucho más modesta, limitándonos a formular algunas de las que podíamos llamar «hipótesis razonables» (más «cualitativas» que «cuantitativas»), basadas en nuestro conocimiento, aunque sea parcial, de la realidad en la que nos movemos, 
así como en la propuesta de sistematización teórica que acabamos de resumir. Consideraciones y propuestas que esperamos puedan servirnos de guía para el desarrollo de algunas investigaciones específicas a abordar en los próximos meses.

Algunos problemas de tipo general

El primer aspecto a considerar, en relación con las preguntas anteriormente formuladas es que, tras el proceso general iniciado en $1984(3 ; 32 ; 132 ; 133)$, la atención en salud mental se organiza en Andalucía sobre la base formal de un modelo comunitario del tipo del que hemos caracterizado anteriormente. La desaparición de los hospitales psiquiátricos públicos (32), la existencia de una nueva red sanitaria especializada en salud mental, su territorialización e integración plena en el sistema sanitario público, el desarrollo de una red de recursos de apoyo social desde una Fundación pública intersectorial (FAISEM) y la existencia en los distintos dispositivos de equipos multiprofesionales, concuerdan básicamente con nuestra exposición y con la referencia explícita a los principios comunitarios que aparece en distintos documentos oficiales, desde la Ley de creación del IASAM en 1984 (3; 132) hasta el vigente Plan Andaluz de Salud Mental (134).

En relación con los temas de rehabilitación y apoyo social hay que hacer referencia, también positiva desde nuestro punto de vista, a la existencia en la red de dispositivos específicos para la rehabilitación y el apoyo social. Por un lado las unidades de rehabilitación, que se sitúan claramente dentro del sistema sanitario; $\mathrm{y}$, por otro, dispositivos y programas específicos de apoyo social, diversificados y gestionados, como hemos dicho, desde una estructura pública e intersectorial. Estos últimos se encargan básicamente de los aspectos que anteriormente denominamos «instrumentales» y se coordinan con los servicios sanitarios a través de una estructura de ámbito provincial. Coordinación que plantea todavía bastantes problemas en su funcionamiento cotidiano, entre otras cosas por la diferente estructura territorial de una y otra red.

Pero además, sin infravalorar en absoluto la trascendencia histórica y las favorables consecuencias de este cambio sobre muchos y significativos aspectos de la atención en salud mental $(3 ; 133)$, en relación con la atención comunitaria, la rehabilitación y el apoyo social a personas con trastorno mental severo, hay que hacer referencia a otros varios tipos de problemas prácticos. Aunque su delimitación cuantitativa requeriría como ya hemos dicho actividades de investigación, dada la escasez de información fiable y detallada sobre aspectos importantes del funcionamiento de los servicios, creemos que gran parte de ellos son fácilmente 
evidenciables, al menos, como una primera aproximación al tema. Planteándolos, por tanto, como meras «hipótesis razonables», que habría que confirmar y definir cuantitativamente en el futuro, pensamos que es necesario considerar al menos tres grandes bloques de problemas que, aunque están claramente interrelacionados, pueden y deben individualizarse.

En primer lugar y ello tiene que ver con el papel de la filosofía y la estrategia de rehabilitación en la atención a las personas con trastornos mentales graves, consideramos necesario hablar de la insuficiente dedicación que todavía siguen recibiendo estas personas, en lo referido al tipo y número de intervenciones que caracterizan habitualmente su atención. Además de sospechar una cobertura incompleta, si relacionamos las cifras de población atendida $(134 ; 135)$ con la extrapolación de prevalencias poblacionales de países con mejores datos epidemiológicos $(33 ; 37 ; 61)$, la comparación de la escasa información publicada sobre Andalucía (10; 136-138), con la de otros países europeos e incluso con la de otras regiones españolas $(12 ; 136)$, ofrece una imagen de la situación que debemos calificar, en relación con el planteamiento teórico que defendemos, como notoriamente deficiente. Imagen que, además de concordar con el conocimiento directo que tenemos, indica que la atención habitual, en contra de lo que se desprende del consenso profesional internacional anteriormente resumido, se limita a la atención intensiva y puntual en las crisis, a un tratamiento casi exclusivamente farmacológico y a revisiones de periodicidad bimensual, en las que se hace poco más que controlar la medicación. Tampoco el uso de dispositivos más específicos como las unidades de rehabilitación (135), que podría compensar las carencias de la atención en los equipos, o, a otro nivel, el de los recursos de apoyo social (139), afecta, pese al incremento continuado de cobertura, a la mayoría de la población afectada. Es verdad que las diferencias entre las recomendaciones técnicas y la práctica asistencial son comunes en otros contextos $(137 ; 140-142)$, pero la «brecha» (142) sigue siendo en nuestro caso todavía importante.

En segundo lugar y también como medida indirecta de la importancia real de la rehabilitación, entendida como filosofía y estrategia, aunque es aún más difícil de cuantificar, creemos que está justificado hablar de algo así como el «déficit comunitario» de los servicios, concepto «cualitativo» que traducen insuficientemente diferentes indicadores cuantitativos como son, por ejemplo, el escaso número de intervenciones fuera del espacio físico de los equipos de Salud Mental -menos del 8\% del total, según los datos oficiales de 2004 (135)-, o, a un nivel muy diferente, la frecuencia de «prácticas restrictivas» (entre ellas el anteriormente denominado «amarre», hoy púdicamente disfrazado de «contención mecánica») en espacios de hospitalización. Y también, aunque en términos menos medibles, el uso, por un lado, de unidades de media estancia o estructu- 
ras residenciales y, por otro, de talleres ocupacionales, como espacios de «depósito» $\mathrm{o}$ «aparcamiento» ${ }^{9}$ de personas con «dificultades de manejo» (entendiendo por tales las que no pueden «manejarse» con fármacos y visitas de revisión bimensuales). Tampoco son fácilmente medibles las pautas habituales de trabajo en equipo, pero no parece difícil detectar funcionamientos poco compatibles con los principios de la atención comunitaria, en términos de corporativismo profesional y tendencias complementarias a reestablecer o reforzar las jerarquías tradicionales («mando único del psiquiatra»), por una parte, y a generar líneas de trabajo «autónomo» y fuera de los equipos, por otra ${ }^{10}$. Las opiniones de los usuarios y usuarias tampoco reflejan, en las pocas ocasiones en que se han recogido, una gran satisfacción con estos aspectos, incluido el trato recibido por bastantes profesionales, aspecto este último traducido al «lenguaje políticamente correcto» como «trato amable», pero «con una fuerte variabilidad entre los profesionales» (143).

Y, finalmente, lo que podemos llamar «paradoja de la rehabilitación», referida al hecho de ser las intervenciones englobables en los programas tanto de rehabilitación como de apoyo social no residencial (especialmente el relacionado con el empleo) las que presentan un déficit más llamativo (135; 139). Situación que contrasta con el hecho conocido (aunque no siempre fácilmente cuantificable con el actual sistema de información, con poca capacidad todavía para darnos información precisa sobre este y otros aspectos de la atención a este colectivo) de que más de una de las actuales unidades de rehabilitación tienen una evidente infrautilización en términos cuantitativos, cuando no realizan funciones (tratamientos farmacológicos, «psicoterapias», etc.) de encaje discutible dentro del perfil funcional con el que se crearon (132) y para cuyo desarrollo la red cuenta, al menos teóricamente, con otro tipo de dispositivos. Del mismo modo, situándonos en los recursos ocupacionales y laborales, y pese a una dotación todavía insuficiente, comparada con sus potenciales beneficiarios, no es extraño encontrarse con dificultades para completar las plazas disponibles.

El tema de las unidades de rehabilitación puede verse como sintomático de una determinada manera de enfocar la discusión. Aunque su funcionamiento no ha sido evaluado sistemáticamente y hay, a simple vista, funcionamientos muy variables entre unas y otras, circula de vez en cuando el «rumor» (del que hasta

9 Todavía sigue siendo posible encontrarse, en un «informe» de derivación de un equipo o toda una área de salud mental, con la doble indicación: «necesita Casa Hogar o Comunidad Terapéutica», nos imaginamos que en función de lo que tienen en común, es decir «la lejanía», ya que no sus funciones, como es obvio, bastante diferentes.

10 También aquí y como indican los análisis sistémicos, cada uno tiende a puntuar las secuencias de interacción según su conveniencia. 
el momento no tenemos ninguna evidencia documental) de que, a distintos niveles de la estructura del sistema, algunos parecen pretender buscar la solución para las que no funcionan cerrándolas todas. $\mathrm{O}$, como alternativa aparentemente menos traumática, integrándolas en hospitales de día, una figura a la que tenemos la impresión de que a veces se le atribuye un prestigio un tanto mítico, a pesar de los resultados de evaluaciones internacionales, mucho más cautos con respecto a su utilidad y a la posible unificación de los distintos tipos de programas llamados «de día» $(144 ; 145)$. Parece razonable pensar que una decisión de estas características, a nivel central o local, debería plantearse previa evaluación de la adecuación de las unidades a sus objetivos iniciales ${ }^{11}$, incluyendo el análisis concreto de lo que funciona bien y mal en cada una de ellas, algo que hasta el momento no se ha hecho, al menos públicamente. Pero sería preciso también valorar las ventajas e inconvenientes de unificar dos tipos de programas muy diferentes en cuanto a usuarios e intervenciones y, en todo caso, garantizar, en aquellas situaciones en que pudiera tener sentido su unificación (zonas rurales de población escasa, dispersa y con difícil acceso a servicios más centralizados), que los programas de rehabilitación van a ponerse en marcha y/o mantenerse dentro del indiferenciado marco del nuevo dispositivo. Si no, podría producirse otra paradoja: la de que no habiendo sido capaces de hacer funcionar dichos programas en una unidad de rehabilitación concreta, pensemos que, más o menos con el mismo personal, vamos a conseguirlo ahora en un entorno más complicado todavía, básicamente por el efecto mágico de cambiar el nombre del dispositivo.

En conjunto, parece que la situación de la «rehabilitación» en sus diversos sentidos deja todavía bastante que desear en nuestro sistema comunitario de atención en salud mental. Y ello sin entrar en problemas más concretos de calidad en ambas redes, y sin olvidar que hay excepciones evidentes, aunque no siempre adecuadamente difundidas, en todos y cada unos de estos aspectos, en determinadas Áreas, Unidades y profesionales. Pero el cuadro general no parece ser muy diferente del aquí señalado y nos obliga a afinar el diagnóstico y a intentar establecer estrategias de mejora, que nos permitan, entre otras cosas, rentabilizar mejor los esfuerzos que distintos sectores y agentes implicados vienen (venimos) realizando hasta ahora.

11 Y que era (132), no lo olvidemos, tanto facilitar la aplicación de programas específicos de rehabilitación, en los casos más complejos, cuanto impulsar el desarrollo de planteamientos y técnicas rehabilitadoras en el conjunto de la red, dado el evidente subdesarrollo que, al menos, al inicio, había al respecto (cuando «la rehabilitación»o «no servía para nada» o «la veníamos haciendo todos desde siempre»). 
Dos grandes tipos de posibles factores condicionantes

En el origen de esos problemas parece haber, como siempre, al menos dos tipos de factores causales: uno referido a la cantidad de recursos y otro a su funcionamiento, adelantando la hipótesis general de que aunque los recursos son todavía insuficientes, también es cierto que hay un margen de uso racional desaprovechado, por razones organizativas pero también de orientación y formación de los profesionales.

Es verdad que una parte de los problemas tiene que ver con la dotación actual de los distintos servicios de atención. A este respecto hay que hacer referencia a dos temas, uno histórico y otro actual. El primero guarda relación con el desarrollo de servicios derivado de la Reforma Psiquiátrica $(3 ; 133)$, que si bien ha cumplido de manera satisfactoria, en nuestra opinión, sus objetivos iniciales (unificar recursos, cambiar el modelo de servicios, integrar la atención sanitaria en el Sistema Sanitario público y articular un sistema de gestión de recursos intersectoriales de apoyo social), ha cubierto sólo parcialmente otros dos importantes aspectos: la puesta al día en términos cuantitativos de los recursos materiales y humanos que nos permitan, al menos, una homologación razonable con el entorno europeo en el que nos integramos, y la generalización de prácticas comunitarias y de calidad en los distintos dispositivos de la nueva red. Del segundo aspecto hemos hablado ya en el apartado anterior («déficit comunitario») y volveremos a insistir en él un poco más adelante. Por su parte, el primero, en su visión no sólo histórica sino actual, puede constatarse comparando datos de recursos materiales y humanos (135) con los de distintos países $(1 ; 12 ; 120 ; 137 ; 146)$, o de otras regiones españolas $(2 ; 119 ; 136)$, incluso descontando el efecto diferenciador que tiene en muchos de ellos las obvias diferencias económicas y sociales (ingresos totales, gasto sanitario y social por habitante, papel de las estructuras familiares, plazas hospitalarias previas, etc.) y sin perder la perspectiva del importante progreso realizado en estos años.

Pero no es éste ni el único ni, en nuestra opinión, el principal factor que explica la mayoría de los problemas mencionados, especialmente los dos últimos («déficit comunitario» y «paradoja de la rehabilitación»). Para nosotros, el aspecto más importante, sin cuya solución de poco servirá incrementar los recursos, que corren el riesgo de seguir siendo mal utilizados, tiene que ver con el funcionamiento actual de los distintos servicios, especialmente en lo referido a la atención que reciben las personas con graves problemas de salud mental. Problemas de funcionamiento que implican tanto a profesionales como a gestores, sobre una base común, aunque evidentemente con matices diferenciales: la diversidad de orientaciones y el todavía importante grado de desconocimiento que, en unos y otros y 
en mayor medida de lo debido, sigue rodeando lo relacionado con la atención comunitaria en general y con la rehabilitación (en sus distintos aspectos o niveles de uso) y el apoyo social, en salud mental. Aspectos que, más allá de la relativa escasez de recursos, explicarían la pauta habitual de intervención, incluyendo las escasas derivaciones a servicios de rehabilitación y apoyo social no residencial. De hecho, esa pauta es consistente con una visión «tradicional» de la atención, centrada en el control de síntomas y el manejo puntual de descompensaciones mediante intervenciones básicamente farmacológicas, frente a la visión rehabilitadora de apoyar a las personas para una vida activa en la comunidad, que exige, como hemos intentado mostrar, un trabajo muy diferente así como muchos otros tipos de técnicas. Pauta «habitual» que, sin embargo, no es siempre la única practicada, existiendo territorios, dispositivos y profesionales que muestran, en su trabajo cotidiano, que el único factor a tener en cuenta para explicar los problemas no es la escasez de recursos.

Algunas estrategias para intentar mejorar la situación

Para finalizar, teniendo en cuenta lo anterior y sin pretender «inventar la pólvora», parece que cualquier propuesta de solución debería coordinar cuidadosamente varias estrategias. Estrategias orientadas lógicamente a modificar los factores condicionantes de los problemas señalados, interviniendo sobre la dotación, estructura y funcionamiento de la red, incluyendo sus distintos niveles de responsabilidad administrativa y profesional.

1. Dado que hay muchos aspectos poco conocidos de la situación, parece necesario un esfuerzo para conocerla, lo que implica mejorar los sistemas de información (SISMA-DIRAYA y Sistema de Información de FAISEM), además de invertir en investigación y difusión de la información.

2. Aunque no sea el único problema, ni baste por sí mismo para solucionar los que hay, parece evidente que poco se puede hacer sin seguir ampliando de manera significativa la actual dotación de recursos, intentando aproximarla a la de otros países de nuestro entorno, en mucho mejores condiciones de atender razonablemente a las personas con trastorno mental severo. Aunque el análisis detallado de necesidades va bastante más allá de nuestras posibilidades, sí queremos señalar nuestra impresión de que es básico incrementar la dotación y capacidad de intervención, al menos, de Equipos Comunitarios, Unidades de Rehabilitación y programas de apoyo social, continuando el impulso del actual Plan Andaluz de 
Salud Mental (134). Además de poner en marcha programas «nuevos» como los de seguimiento intensivo de pacientes con dificultades de atención o los de intervención precoz en psicosis. Y de hacer progresivamente más «igualitaria» e integrada la composición multiprofesional de los equipos.

3. Pero el incremento de recursos serviría de poco si no realizase en el contexto de un impulso real y sostenido al cambio de orientación en el funcionamiento de la red y sus distintos componentes. Ello implica medidas organizativas que integren efectivamente, en la gestión general del Sistema Sanitario, verdaderos Servicios Comunitarios de Salud Mental, asegurando su funcionamiento como tales mediante las necesarias medidas de orientación (guías clínicas, metodología de procesos asistenciales) ${ }^{12}$, formación, incentivación (no sólo vía retribuciones, sino también con participación) y control de calidad. Y, recíprocamente, medidas que mejoren la adecuación y funcionamiento de los recursos dependientes de FAISEM y su coordinación con los sanitarios, incluyendo una distribución territorial más homogénea.

4. Pero todo ello exige también trabajo de sistematización teórica, investigación y experimentación científica y debate profesional y ciudadano. Aspectos que no deben separarse del esfuerzo por adoptar mecanismos específicos de gestión como puede ser los procedimientos de gestión clínica, justamente enfatizados actualmente. Pero esa necesidad de integrar ambos aspectos es clave, en el doble sentido de que los mecanismos de gestión pueden facilitar las modificaciones funcionales, pero no sustituyen la necesidad de definir claramente las orientaciones generales (valores y estrategias) y las técnicas concretas de intervención, en el marco de una atención comunitaria en salud mental coherente y tecnológicamente adecuada $^{13}$.

Aspecto este último que, en nuestra opinión, implica de modo fundamental a los profesionales agrupados en la AEN, ya que consideramos que nuestra asociación debería seguir siendo un elemento impulsor de la atención comunitaria, en la perspectiva tecnológica que hemos planteado.

12 En ese sentido, el proceso asistencial integrado trastorno mental grave, a punto de publicarse y básicamente coincidente con las líneas expuestas a lo largo de este artículo, puede suponer un impulso razonable en la atención a este colectivo.

13 Es obvio, por ejemplo, que los procedimientos de gestión clínica son potencialmente aplicables en diferentes sistemas y tipos de servicios, incluso en un hospital psiquiátrico. 
Lo que exigiría también entre otras cosas que, además del debate de ideas, hubiese un creciente número de publicaciones en las que los, en absoluto menospreciables, equipos y profesionales que, en distintos dispositivos de la red, hacen hoy verdadera atención comunitaria, rehabilitación y apoyo social en nuestra comunidad autónoma, difundiesen su trabajo y sometiesen a discusión sus experiencias y reflexiones críticas.

\section{BIBLIOGRAFÍA}

(1) «Rehabilitación psicosocial del trastorno mental severo. Situación actual y recomendaciones», Madrid, AEN, 2002, Cuadernos técnicos, 6.

(2) GISBERT, C. (ed.), «Rehabilitación psicosocial y tratamiento integral del trastorno mental», Madrid, AEN, 2003.

(3) LÓPEZ, M., «Luces y sombras de la reforma psiquiátrica andaluza», Hipatia, 2000, 7, pp. 4-8.

(4) LÓPEZ, M., «Rehabilitación y red asistencial. Sistemas de atención a la "cronicidad" en salud mental», en Verdugo, M. A.; LóPEZ, D., y otros, Rehabilitación de salud mental. Situación y perspectivas, Salamanca, Amaru, 2002, pp. 75-105.

(5) LÓPEZ, M.; LARA, L.; LAVIANA, M., «La formación básica en rehabilitación y apoyo social para la atención comunitaria en salud mental», en EsPino, A.; OlABARRÍA, B., La formación de los profesionales de la salud mental en España, Madrid, AEN, 2003, pp. 269-301.

(6) LaViana, M., «La atención a las personas con esquizofrenia y otros trastornos mentales graves desde los servicios públicos: una atención integral e integrada en un modelo comunitario», Apuntes de Psicología (en prensa).

(7) LóPEZ, M., y otros, «Los programas residenciales para personas con trastorno mental severo. Revisión y propuestas», Archivos de Psiquiatría, 2004, 67, 2, pp. 101-128.

(8) LóPEZ, M., y otros, «Actividad productiva y empleo de personas con trastorno mental severo. Algunas propuestas de actuación basadas en la información disponible», Revista de la AEN, 2004, XXIV, 89, pp. 31-65.

(9) LóPEZ, M., y otros, «Evaluación del programa residencial para personas con trastorno mental severo en Andalucía (I): descripción general del programa y del estudio», Rehabilitación Psicosocial, 2005, 2, 1, pp. 2-15.

(10) LÓPEZ, M., y otros, «Evaluación del programa residencial para personas con trastorno mental severo en Andalucía (III): características sociodemográficas, clínicas y de uso de servicios de los residentes», Rehabilitación Psicosocial, 2005, 2, 1, pp. 28-39.

(11) Aparicio, V., «Política asistencial en España: presente y futuro», en Aldaz, J. A.; VÁzQueZ, C. (eds.), Esquizofrenia: fundamentos psicológicos y psiquiátricos de la rehabilitación, Madrid, Siglo XXI, 1996, pp. 293-306.

(12) Salvador-Carulla, L., y otros, «La salud mental en España: Cenicienta en el País de las Maravillas», en Informe SESPAS 2002, Invertir en salud. Prioridades en Salud Pública, Valencia, EVES, 2002, pp. 301-326.

(13) SARraceno, B., La fine dell'Intrattenimento, Milán, Etas Libri, 1995.

(14) Quintanilla, M. A., Tecnología: un enfoque filosófico, Madrid, FUNDESCO, 1989.

(15) Stroll, A., La filosofía analítica del siglo XX, Madrid, Siglo XXI, 2002. 
(16) GonzÁlez, M. I.; López, J. A.; Luján, J. L., Ciencia, Tecnología, Sociedad, Madrid, Tecnos, 1996.

(17) Quintanilla, M. A., «La tecnología como paradigma de acción racional», Revista de Occidente, 2000, 228, pp. 53-74.

(18) Tizón, J. L., «Los nuevos modelos asistenciales en medicina: una visión a partir de algunos de sus fundamentos psicológicos y epistemológicos», Revisiones en Salud Pública, 1995, 4, pp. 57-83.

(19) Álvarez, S., «Racionalidad y método científico», en Olivé, L. (ed.), Racionalidad epistémica. Enciclopedia Iberoamericana de Filosofía, Madrid, Trotta, 1995, pp. 147-169.

(20) DraKe, R. E., y otros, «Implementing Evidence-based Practice in Routine Mental Health Service Settings», Psychiatric Services, 2001, 52, 2, pp. 179-182.

(21) MAYORAL, F., «Rehabilitación psicosocial. Hacia una práctica basada en la evidencia», en RIVAS, F. (ed.), Las psicosis en la comunidad, Madrid, AEN, 2000, pp. 243-262.

(22) ECHEverría, J., Ciencia y valores, Barcelona, Destino, 2002.

(23) ECHEVERRÍA, J., Introducción a la metodología de la ciencia. La filosofía de la ciencia en el siglo XX, Madrid, Cátedra, 2003.

(24) KHUN, T. S., La estructura de las revoluciones científicas, México, FCE, 1971.

(25) LAKATOS, I., La metodología de los programas de investigación científica, Madrid, Alianza, 1989.

(26) Breakey, W. R. (ed.), Integrated Mental Health Services. Modern Community Psychiatry, Nueva York, Oxford University Press, 1996.

(27) Desviat, M., La Reforma Psiquiátrica, Madrid, Dor, 1994.

(28) García, J.; Espino, A.; LARA, L. (eds.), La psiquiatría en la España de fin de siglo, Madrid, Díaz de Santos, 1998.

(29) GonZÁlez DE ChÁveZ, M., La transformación de la asistencia psiquiátrica, Madrid, Mayoría, 1983.

(30) LefF, J. (ed.), Care in the Community: Illusion or Reality?, Londres, Wiley, 1997.

(31) Thornicroft, G.; Szmukler, G. (eds.), Textbook of Community Psychiatry, Oxford University Press, 2001.

(32) LóPez, M.; Laviana, M.; García-Cubillana, P., «Los hospitales psiquiátricos en la(s) reforma(s). Notas para orientar una investigación necesaria», en PÉREZ, F. (coord.), Dos décadas tras la reforma psiquiátrica, Madrid, AEN, 2006, pp. 237-273.

(33) Ruggeri, M., y otros, «Definition and Prevalence of Severe and Persistent Mental Illness», British Journal of Psychiatry, 2000, 177, pp. 149-155.

(34) SHEPHERD, G., «Avances recientes en rehabilitación psiquiátrica», en AldAZ, J.; VÁzQueZ, C. (eds.), Esquizofrenia: fundamentos psicológicos y psiquiátricos de la rehabilitación, Madrid, Siglo XXI, 1996, pp. 1-22.

(35) WING, J. K.; Brown, G. B., Institutionalism and Schizophrenia, Londres, Cambridge University Press, 1970.

(36) Harding, M.; Zubin, J.; Strauss, J. S., «Chronicity in Schizophrenia: Fact, Partial Fact or Artefact?», Hospital and Community Psychiatry, 1987, 38 (5), pp. 477-486.

(37) Parabiaghi, A., y otros, «Severe and Persistent Mental Illness: a Useful Definition for Prioritizing Community-based Health Service Interventions», Social Psychiatry and Psychiatric Epidemiology, 2006, 41, pp. 457-463.

(38) Frances, A.; Pincus, H. A., First MB. The Global Assessment of Functioning Scale (GAF) Diagnostic and Statistical Manual of Mental Disorders-IV, Washington, American Psychiatric Association, 1994. 
(39) AldAZ, J. A.; VÁZQUEZ, C. (eds.), Esquizofrenia: fundamentos psicológicos y psiquiátricos de la rehabilitación, Madrid, Siglo XXI, 1996.

(40) BARton, R., «Psychosocial Rehabilitation Services in the Community Support Systems: a Review of Outcomes and Policy Recommendations», Psychiatric Services, 1999, 50, 4, pp. 525534.

(41) Flexer, R. W.; Solomon, P. L. (eds.), Psychiatric Rehabilitation in Practice, Andover, Stoneham, 1993.

(42) Liberman, R. L., Rehabilitación integral del enfermo mental crónico, Barcelona, Martínez Roca, 1993.

(43) Rebolledo, S. (coord.), Rehabilitación Psiquiátrica, Universidade de Santiago de Compostela, 1997.

(44) RodríGUEZ, A. (coord.), Rehabilitación psicosocial de personas con trastornos mentales crónicos, Madrid, Pirámide, 1997.

(45) WatTs, F. N.; BennetT, D. (eds.), Rehabilitación psiquiátrica. Teoría y práctica, México, Limusa, 1990.

(46) Fakhoury, W.; Priebe, S., «The Process of Deinstitutionalization: an International Overview», Current Opinion in Psychiatry, 2002, 15, pp. 187-192.

(47) Ancil, R. J.; Holliday, S.; HiggenbtTam, J. (eds.), Schizophrenia. Exploring the Spectrum of Psychosis, Chichester, Wiley, 1994.

(48) CiomPI, L., "Affect-logic: an Integrative Model of the Psyche and its Relations to Schizophrenia», British Journal of Psychiatry, 1994, 164, pp. 51-55.

(49) Zubin, J.; SpRing, B., «Vulnerability: a New View of Schizophrenia», Journal of Abnormal Psychology, 86, pp. 103-126.

(50) GREEN, M. F., «What Are the Functional Consequences of Neurocognitive Deficits in Schizophrenia?», American Journal of Psychiatry, 1996, 153, pp. 321-330.

(51) Zandio-Zorrilla, M., y otros, «Alteraciones cognitivas en la esquizofrenia», Medicina Clínica, 2005, 6, 5, pp. 17-26.

(52) Muesser, K. T.; TARrier, N., Handbook of Social Functioning in Schizophrenia, Boston, Allyn and Bacon, 1998.

(53) ZubIN, J., «Suiting Therapeutics Intervention to the Scientific Models of Aetiology», British Journal of Psychiatry, 1989, 155, pp. 9-14.

(54) OMS, Clasificación Internacional del funcionamiento, de la discapacidad y de la salud, Madrid, IMSERSO, 2001.

(55) VÁzquez-BARquero, J. L., y otros, «La discapacidad: modelos interpretativos y su influencia en el nuevo sistema de clasificación de discapacidades de la Organización Mundial de la Salud», Archivos de Psiquiatría, 2000, 63, 1, pp. 5-20.

(56) Lastra, I.; Martínez-Chamorro, M. J. y Grupo Cantabria en Discapacidades, «Funcionamiento, discapacidad y rehabilitación», Informaciones Psiquiátricas, 2004, 175, pp. 19-30.

(57) Warner, R., Recovery from Schizophrenia. Psychiatry and Political Economy, Londres, Brunner-Rutledge, 2003.

(58) Harding, C. M., «An Examination of the Complexities in the Measurement of Recovery in Severe Psychiatric Disorders», en Ancil, R. J.; Holliday, S.; HiggendtTaM, J. (eds.), Schizophrenia. Exploring the Spectrum of Psychosis, Chichester, Wiley, 1994, pp. 153-168.

(59) Jacobson, N.; Greenley, D., «What is Recovery? A Conceptual Model and Explication», Psychiatric Services, 2001, 52, pp. 482-485. 
(60) McLeAn, A. H., «From Ex-patient Alternatives to Consumer Options: Consequences of Consumerism for Psychiatric Consumers and Ex-patient Movement», International Journal of Health Services, 2000, 30, 4, pp. 821-847.

(61) Bromet, E. J.; DeW, M. A.; Eaton, W. W., «Epidemiology of Psychosis with Special Reference to Schizophrenia», en Tsuang, M. T.; TohEn, M. (eds.), Textbook in Psychiatric Epidemiology, Nueva York, Wiley, 2002, pp. 365-388.

(62) HARRISON, G., y otros, «Recovery from Psychotic Illness: a 15 and 25 Year International Follow-up Study», British Journal of Psychiatry, 2001, 178, pp. 506-517.

(63) MCLEAN, A., «Empowerment and the Psychiatric Consumer/ex-patient Movement in the United States: Contradictions, Crisis and Change», Social Science and Medicine, 1995, 40, 8, pp. 1.053-1.071.

(64) Anthony, W. A., «A Recovery-oriented Service System: Setting Some System Level Standards», Psychiatric Rehabilitation Journal, 2000, 24 (2), pp. 159-168.

(65) Bellack, A. S., «Scientific and Consumer Models of Recovery in Schizophrenia: Concordance, Contrasts and Implications», Schizophrenia Bulletin, 2006, 32, 3, pp. 432-442.

(66) DAvidson, L., y otros, «Simply To Be Let In»: Inclusion as a Basis for Recovery», Psychiatric Rehabilitation Journal, 2001, 24, pp. 375-388.

(67) CoRrigan, P. W.; Penn, D., «Disease and Discrimination: Two Paradigms that Describe Severe Mental Illness», Journal of Mental Health, 1997, 6, 4, pp. 355-366.

(68) Lester, H.; GASK, L., «Delivering Medical Care for Patients with Serious Mental Illness or Promoting a Collaborative Model of Recovery», British Journal of Psychiatry, 2006, 188, pp. 401-402.

(69) LARA, L.; LóPEZ, M., La provisión de recursos de apoyo social en Andalucía, en GARCía, J.; EsPinO, A.; LARA, L. (eds.), op. cit., 1998, pp. 213-224.

(70) Gaebel, W., y otros, «Schizophrenia Practice Guidelines: International Survey and Comparison», British Journal of Psychiatry, 2005, 187, pp. 248-255.

(71) Alanen, Y. O., Schizophrenia. Its Origins and Need-adapted Treatment, Londres, Karnack Books, 1997.

(72) American Psychiatric Association, Practice Guidelines for the Treatment of Patients with Schizophrenia, Nueva York, APA, 2004.

(73) Lehman, A. F., y otros, «The Schizophrenia Patient Outcome Research Team (PORT): Updated Treatment Recommendations 2003», Schizophrenia Bulletin, 2004, 30, 2, pp. 193-217.

(74) National Institute for Clinical Excellence (Nice), «Schizophrenia: Core Interventions in the Treatment and Management of Schizophrenia in Primary and Secondary care», Clinical Guidelines, 1, 2002.

(75) AgÈnCIA D’AVAluació de teCNOlogia I Ricerca MÈDIQUes, «Guía de práctica clínica para la atención al paciente con esquizofrenia. Versión para la aplicación en la práctica clínica», Barcelona, 2004.

(76) CAldas, J. M.; Torres, F., Atención comunitaria a personas con trastornos psicóticos, Washington, Organización Panamericana de la Salud, 2005.

(77) FARKAS, M., «Avances en rehabilitación psiquiátrica: una perspectiva norteamericana», en AldaZ, J. A.; VÁZQuez, C. (eds.), op. cit., 1996, pp.167-185.

(78) Mueser, K. T.; Drake, R. E.; Bond, G. R., «Recent Advances in Psychiatric Rehabilitation for Patients with Severe Mental Illness», Harvard Review of Psychiatry, 1997, 5, pp. 123-137.

(79) Wallace, C. J., «Psychiatric Rehabilitation», Psychopharmacological Bulletin, 1993, 29 (4), pp. 537-548. 
(80) Rivas, F. (ed.), Las psicosis en la comunidad, Madrid, AEN, 2000.

(81) Fenton, W. S., «Involving Perspectives on Individual Psychotherapy for Schizophrenia», Schizophrenia Bulletin, 2000, 26 (1), pp. 47-72.

(82) Hogarty, G. E., y otros, «Three-year Trials of Personal Therapy Among Schizophrenic Patients Living with or Independent of Family. I: Description of the Study and Effects on Relapse Rates. II: Effects on Adjustment of Patients», American Journal of Psychiatry, 1997, 154, pp. 15041524.

(83) Dickerson, F. B.; Lehman, A. F., «Evidence Based Psychotherapy for Schizophrenia», Journal of Nervous and Mental Disorders, 2006, 194, 1, pp. 3-9.

(84) Jiménez, J. F.; Maestro, J. C.; Moreno, B., «Tratamiento psicológico de la esquizofrenia», Cuadernos de Psiquiatría Comunitaria, 2004, 4, 2, pp. 159-174.

(85) Vallina, O.; Lemos, S., «Tratamientos psicológicos eficaces para la esquizofrenia», Psicothema, 2001, 13, pp. 345-364.

(86) ScotT, J., «Cognitive Therapy of Affective Disorders: a Review», Journal of Affective Disorders, 1996, 37, pp. 1-11.

(87) Perona, S., y otros, «Terapia cognitivo-conductual en la esquizofrenia», Guía clínica, Madrid, Minerva, 2003, 52, 6, pp. 771-779.

(88) Pilling, S., y otros, «Psychological Treatments in Schizophrenia. I: Meta-analysis of Family Intervention and Cognitive Behaviour Therapy», Psychological medicine, 2002, 32, 5, pp. 763-782.

(89) MueSER, K. T., y otros, «Illness Management and Recovery: a Review of the Research», Psychiatric Services, 2002, 53, 10, pp. 1272-1284.

(90) Nose, M., y otros, «Clinical Interventions for Treatment Non-adherence in Psychosis: Meta-analysis», British Journal of Psychiatry, 2003, 183, pp. 197-206.

(91) GiL, F.; LeÓN, J. M.; Jarana, L. (eds.), Habilidades sociales y salud, Madrid, Pirámide, 1995.

(92) Moxley, D. P.; Mowbray, C. T.; Brown, K. S., «Supported Education», en Flexer, R. W.; Solomon, P. L. (eds.), Psychiatric Rehabilitation in Practice, Andover Stoneham, 1993, pp. 137-153.

(93) PILling, S., y otros, «Psychological Treatments in Schizophrenia. II: Meta-analysis of Random-controlled Trials of Social Skills Training and Cognitive Remediation», Psychological medicine, 2002, 32, 5, pp. 783-791.

(94) BREnNER, H. D., y otros, «Treatment of Cognitive Dysfunctions and Behavioural Deficits in Schizophrenia», Schizophrenia Bulletin, 1992, 18, 1, pp. 21-26.

(95) Silverstein, S. M.; WiLkINS, S. M., «At Issue: The Future of Cognitive Rehabilitation of Schizophrenia», Schizophrenia Bulletin, 2004, 30, 4, pp. 679-692.

(96) Twamley, E. W.; Jeste, D. V.; BellaK, A. S., «A Review of Cognitive Training in Schizophrenia», Schizophrenia Bulletin, 2003, 29, 2, pp. 359-382.

(97) Anderson, C.; ReIss, D.; Hogarty, G., Esquizofrenia y familia. Guía práctica de psicoeducación, Buenos Aires, Amorrortu, 1988.

(98) SANZ, F. J.; CAÑIVETE, J., «"Psicoeducación” de familiares de pacientes esquizofrénicos», en AldaZ, J. A.; VÁzQueZ, C. (eds.), op. cit., 1996, pp. 205-226.

(99) LóPEZ, M.; LARA, L., «Guías operativas 8 ("Programa residencial”), 9 ("Programa ocupacional y laboral") y 10 ("Otros programas de apoyo social")», en CALDAS Y TORRES, Atención comunitaria a personas con trastornos psicóticos, Washington, Organización Panamericana de la Salud, 2005, pp. 83-105. 
(100) Carling, P. J., «Housing and Supports for Persons with Mental Illness: Emerging Approaches to Research and Practice», Hospital and Community Psychiatry, 1993, 44 (5), pp. 439-449.

(101) FAKHOURY, W., y otros, «Research in Supported Housing», Social Psychiatry and Psychiatric Epidemiology, 2002, 37, pp. 301-315.

(102) OgILvie, R. J., «The State of Supported Housing for Mental Health Consumers: a Literature Review», Psychiatric Rehabilitation Journal, 1997, 21 (2), pp. 1222-131.

(103) Bond, G. R., y otros, «Implementing Supported Employment as an Evidence-Based Practice», Psychiatric Services, 2001, 52 (3), pp. 313-322.

(104) CooK, J.; Razzano, L., «Vocational Rehabilitation for Persons with Schizophrenia: Recent Research and Implications for Practice», Schizophrenia Bulletin, 2000, 26, 1, pp. 87-103.

(105) Crowther, R., y otros, «Helping People with Severe Mental Illness to Obtain Work: a Systematic Review», British Medical Journal, 2001, 322, pp. 204-208.

(106) Angermayer, M. C.; Schulze, B., «Reducing the Stigma of Schizophrenia: Understanding the Process and Options for Interventions», Epidemiologia e Psichiatria Sociale, 2001, 10,1, pp. 1-7.

(107) LóPEZ, M., y otros, «La lucha contra el estigma y la discriminación en salud mental. Una estrategia compleja basada en la información disponible», enviado a Archivos de Psiquiatría, 2006.

(108) U. S. Department of Health Human Services, Mental Health: A Report of the Surgeon General, National Institute of Mental Health, 1999.

(109) Burns, T., Community Mental Health Teams. A guide to Current Practices, Oxford University Press, 2004.

(110) Thornicroft, G.; Tansella, M., The Mental Health Matrix: a Manual to Improve Services, Cambridge University Press, 1999.

(111) Jenkins, R.; FIELD, V., The Primary Care of Schizophrenia, Londres, HMSO, 1996.

(112) Fenton, W., y otros, «A Randomised Trial of General Hospital Versus Residential Alternative Care for Patients with Severe and Persistent Mental Illness», American Journal of Psychiatry, 1998, 155, pp. 516-522.

(113) Mosher, L., «Soteria and Other Alternatives to Acute Psychiatric Hospitalisation: A Personal and Professional Review», Journal of Nervous and Mental Diseases, 1999, 187, pp. 142-149.

(114) SHEPHERD, G., «The Ward-in-a-house: Residential Care for the Severely Disabled», Journal of Mental Health, 1995, 31, pp. 53-69.

(115) World Health Organization, Organization of Services for Mental Health, Ginebra, Who, Mental Health Policy and Service Guidance Package, 2003.

(116) HERnÁNDEZ, M., «Los programas de seguimiento intensivo en la comunidad: una alternativa a la hospitalización ¿y algo más?», en RIVAS, F., 2000, pp. 131-189.

(117) Simmonds, S., y otros, «Community Mental Health Team Management in Severe Mental Illness: a Systematic Review», British Journal of Psychiatry, 2001, 178, pp. 497-502.

(118) World Health Organization, Planning and Budgeting to Deliver Services for Mental Health, Ginebra, Who, Mental Health Policy and Service Guidance Package, 2003.

(119) PÉREZ, F. (coord.), Dos décadas tras la reforma psiquiátrica, Madrid, AEN, 2006.

(120) BECKER, T., y otros, «Provision of Services for People with Schizophrenia in Five European Regions», Social Psychiatry and Psychiatric Epidemiology, 2002, 37, pp. 465-474.

(121) FUNDACIÓN ANDALUZA PARA LA INTEGRACIÓN SOCIAL DEL ENFERMO MENTAL, «Criterios para el desarrollo del programa residencial», Sevilla, FAISEM, Documentos de Trabajo, 1, 2005. 
(122) FUNDACIÓN ANDALUZA PARA LA INTEGRACIÓN SOCIAL DEL ENFERMO MENTAL, «Criterios para el desarrollo del programa ocupacional-laboral», Sevilla, FAISEM, Documentos de Trabajo, 2, 2005.

(123) VuORI, H., «Qué es la Atención Primaria de Salud», Atención Primaria, 1983, pp. 3-4.

(124) Saraceno, B., «Psychosocial Rehabilitation as a Public Health Strategy», Psychiatric Rehabilitation Journal, 1997, 20, 4, pp. 10-15.

(125) Ferrara, F. A.; Acebal, E.; Paganini, J. M., Medicina de la comunidad, Buenos Aires, Intermédica, 1976.

(126) Gittelman, M., «Tertiary Prevention of Mental Disorders», en Thornicroft, G.; Szmukler, G. (eds.), Textbook of Community Psychiatry, Oxford University Press, 2001, pp. 509516.

(127) Fernández de Larrinoa, P.; SÁez de Ibarra, A.; Bulbena, A., «Evaluación clínica en el ámbito de la psiquiatría social y la rehabilitación psiquiátrica», en BULBENA, A.; BERRIOS, G. E.; Fernández de LarrinoA, P. (eds.), Medición clínica en Psiquiatría y Psicología, Barcelona, Masson, 2000, pp. 509-544.

(128) Wallace, C. J., «Functional Assessment in Rehabilitation», Schizophrenia Bulletin, 1986, 12, pp. 604-630.

(129) Bigelow, D., «Supportive Homes for Life Versus Treatment Way-stations: an Introduction to TAPS Project 41», Community Mental Health Journal, 1998, 34, 4, pp. 403-405.

(130) Grove, B., y otros, The Social Firm Handbook, Brighton, Pavilion, 1997.

(131) LÓPEZ, M.; ÁlVAREZ, F.; LAVIANA, M., «Empresas sociales y rehabilitación psiquiátrica en Andalucía», Revista de la AEN, 1998, XVIII, 68, pp. 735-747.

(132) Instituto ANDALUZ De SAlUd MENTAL, La reforma Psiquiátrica en Andalucía 19841990, IASAM, Sevilla, 1988.

(133) CARMONA, J., y otros, «Análisis histórico crítico de la reforma psiquiátrica andaluza», en PÉREZ, F. (coord.), Dos décadas tras la reforma psiquiátrica, Madrid, AEN, 2006, pp. 145-198.

(134) CONSEJERÍA DE SAlud, Plan integral de Salud Mental de Andalucía 2003-2007, Servicio Andaluz de Salud, Sevilla, 2003.

(135) Servicio andaluz De Salud, Memoria 2004, sas, Sevilla, 2005.

(136) Montilla, J. F., y otros, «Uso de servicios ambulatorios de salud mental en España. Consumo de recursos en primer año de asistencia a pacientes nuevos», Revista de la AEN, 2002, XXII, 84, pp. 25-47.

(137) Salice, H. J., y otros, «Needs for Care and Effectiveness of Mental Health Care Provision for Schizophrenic Patients in Two European Regions: A Comparison Between Granada (Spain) and Mannheim (Germany)», Acta Psychiatrica Scandinavica, 1999, 100, pp. 328-334.

(138) Moreno, B. (ed.), El registro de casos de esquizofrenia de Granada, Madrid, AEN, 2004.

(139) FUNDACIÓN ANDALUZA PARA LA INTEGRACIÓN SOCIAL DEL ENFERMO MENTAL, Memoria 2005, FAISEM, Sevilla, 2006.

(140) World Health Organization, World Health Report 2001, Mental Health: New Understanding, New Hope, Ginebra, wHO, 2001.

(141) West, J. C., y otros, «Patterns and Quality of Treatment for Patients with Schizophrenia in Routine Psychiatric Practice», Psychiatric Services, 2005, 56, 3, pp. 283-291.

(142) Bravo, M. F.; Díaz, B.; Fernández, A., «La atención a los trastornos mentales graves», en PÉREZ, F. (coord.), Dos décadas tras la reforma psiquiátrica, Madrid, AEN, 2006, pp. 275-296.

(143) PÉrez, C. (coord.), La atención a la salud mental en Andalucía: presente y futuro, Granada, Escuela Andaluza de Salud Pública, 2004. 
(144) Schene, A. H., «The Effectiveness of Psychiatric Partial Hospitalization and Day Care», Current Opinion in Psychiatry, 2004, 17, pp. 303-309.

(145) CATTY, J.; Goddard, K.; BuRns, T., «Social Services Day Care and Health Services Day Care in Mental Health: Do They Differ?», International Journal of Social Psychiatry, 2005, 51, 2, pp. 151-161.

(146) World Health Organization, Mental Health Atlas 2005, Ginebra, WHO, 2005.

* Marcelino López, psiquiatra y sociólogo. Director de programas, evaluación e investigación. FAISEM. Sevilla; Margarita Laviana, psicóloga clínica. Coordinadora de Comunidad Terapéutica. Área de Salud Mental. Hospital «Virgen del Rocío». SAS. Sevilla.

Correspondencia: Marcelino López, FAISEM, Avenida de las Ciencias, 27. Acceso A, 41020 Sevilla, marcelino.lopez@juntadeandalucia.es

** Fecha de recepción: 21-XI-2006. 